\title{
Current instabilities in resonant tunnelling diodes based on GaN/AIN heterojunctions
}

\author{
A.E. Belyaev ${ }^{1,4}$, C.T. Foxon ${ }^{1}$, S.V. Novikov ${ }^{1}$, O. Makarovsky ${ }^{1}$, L. Eaves ${ }^{1}$, M.J. Kappers², \\ J.S. Barnard ${ }^{2}$, C.J. Humphreys ${ }^{2}$, S.V. Danylyuk ${ }^{3}$, S.A. Vitusevich ${ }^{3}$, A.V. Naumov ${ }^{4}$ \\ ${ }^{1}$ School of Physics and Astronomy, University of Nottingham, Nottingham NG7 2RD, UK \\ ${ }^{2}$ Dept. of Materials Science and Metallurgy, University of Cambridge, Cambridge CB2 3QZ, UK \\ ${ }^{3}$ Insitut fur Schichten und Grenzflachen and CNI-Center of Nanoelectronic Systems for Information Technology, \\ Forschungszentrum Julich, Julich 52425, Germany \\ ${ }^{4} V$. Lashkaryov Institute of Semiconductor Physics, National Academy of Sciences of Ukraine \\ 41, prospect Nauky, 03028 Kyiv, Ukraine
}

\begin{abstract}
Present paper studies double barrier resonant tunnelling diodes (DB-RTD) based on GaN/AlGaN heterostructures, grown by plasma-assisted molecular beam epitaxy (PA-MBE). Tunnel (current-voltage, $I-V$ ) and capacitance (capacitance-voltage, $C-V$ ) spectroscopy measurements were performed at the temperature range from 4.2 to $300 \mathrm{~K}$. It has been found that measured characteristics of DB-RTD have complex nonlinear behavior and reveal the current discontinuities of $I-V$ curves. The features can be explained by the existence of polarization fields and interface defects. These effects strongly influence on the potential profile of the DBRTD heterostructures. To understand physics of the processes, numerical simulations of the given structures, using a model based on real-time Green's functions, have been performed. Comparative analysis of experimental and numerical data showed that the current instability and nonlinearity of characteristics of the nitride based DB-RTD can be connected with trapping the electrons onto interfacial and dislocation states in these heterostructures.
\end{abstract}

Keywords: gallium and aluminium nitrides, heterostructure, resonant tunneling diode, capacitance and tunnelling spectroscopy.

Paper received 15.03.04; accepted for publication 17.06.04.

\section{Introduction}

There is an increasingly high level of commercial and scientific interest in nitride semiconductors both nationally and internationally [1]. The group III-nitrides (AlN, GaN and $\mathrm{InN}$ and their solid solutions $\mathrm{AlGaN}$ and $\mathrm{InGaN}$ ) are being used increasingly for amber, green, blue and white light emitting diodes (LEDs) [2], for blue/UV laser diodes (LDs) [3] and for high power, high frequency and high temperature electronic devices (HFETs) [4]. Recently, there has been considerable progress in developing GaN-based devices with lateral transport of carriers. However, very few studies both theoretical and experimental have been performed so far for GaN-based devices exploiting vertical transport of carriers like to resonant tunnelling diodes (RTDs).

Double barrier resonant tunnelling diodes are the basic benchmark quantum tunnelling devices. They have been made in a variety of materials systems, including
(AlGa)AlAs, Si/Ge, and InAs/GaSb. Their main applications are the source of high frequency power operated in millimetre and sub-millimetre range. If RTDs could be produced in the group III-nitride system, a number of novel possibilities exists for development and exploitation. Due to the large band offsets available, it should be possible to observe quantum behaviour at much higher temperatures than in other III-V systems. In practical applications, it might be possible to obtain higher power devices at a given frequency or higher frequency performance at a given power. The enhanced barrier height certainly offers the potential for greater flexibility of design for room temperature operation. If RTDs can be made in Group III-nitrides, a whole new technology could be realised.

Given the attractive scientific and technological nature of GaN/AlGaN RTDs, it is reasonable to ask why there has been so little work on this topic [5-8]. We believe that this is because the material quality in the group 


\section{A.E. Belyaev et al.: Current instabilities in resonant tunnelling diodes based on ...}

III-nitrides has only recently become good enough to attempt to produce such structures. There are two relevant factors: the structural quality of the heterojunctions and the scattering by impurities due to the background doping level. Both factors destroy the translation invariance that leads to the conservation of electron momentum necessary for successful operation of RTDs.

The lattice parameters of the nitrides are much smaller than for other more conventional III-V compounds, with the result that no suitable lattice matched substrate is available. Until recent time, all the structures were grown by hetero-epitaxy on sapphire or SiC substrates. Now, due to the availability of GaN templates, grown by hydride vapour phase epitaxy (HVPE) or metal organic vapour phase epitaxy (MOVPE), the structural and optical properties of GaN films have been dramatically improved. It is very difficult to predict what structural quality will be required for high quality RTD devices, but we note that using homoepitaxy Group III-nitride interfaces comparable with those of the arsenides have now been demonstrated. Impurities should be less of a problem for the nitrides than for the arsenides, because the effective Bohr radius of the impurity is much smaller. The effective mass for $\mathrm{GaN}$ is about 3 times larger than for $\mathrm{GaAs}$, which suggests that quantum wells (QWs) will need to be about 2 times thinner than in arsenides. From our previous experience in arsenides we suggests that GaN well widths of 1 to $4 \mathrm{~nm}$ are needed. For the tunnel barriers the transmission coefficient $(T)$ is given by $T \sim \exp (-k b)$, where $k=\left[2 m^{*}(V-E)\right]^{1 / 2} / \hbar$ is wave-vector of tunnelling electron with effective mass $m^{*}$ and energy $E ; V$ and b are height and width of the barrier. Again from our experience with arsenides this suggests that $\mathrm{AlGaN}$ barrier thickness of 1 to $3 \mathrm{~nm}$ will be appropriate, again sufficiently thick to give every expectation of success. At the same time, we need to remember that the effect of built-in electric field arising due to piezo- and spontaneous polarisation will be very significant in GaN/AlGaN RTD structures. Thus, the main aim of this paper is to investigate the problems of developing the technology of RTDs by plasma-assisted molecular beam epitaxy (PA-MBE).

\section{Experimental details}

The RTD samples used in this study were grown by MBE on Si-doped $\left(n^{+}\right)$, (0001), Ga-polar MOVPE GaN/sapphire templates. The dislocation density in the MOVPE GaN layers was about $2 \cdot 10^{9} \mathrm{~cm}^{-2}$. Active nitrogen for the growth of the group III-nitrides was provided by an $\mathrm{Ox}$ ford Applied Research CARS25 RF activated plasma source. The MBE growth temperature was fixed at $\sim 800^{\circ} \mathrm{C}$. GaN well widths of 1 to $4 \mathrm{~nm}$ with AlN barriers thicknesses of 1 to $3 \mathrm{~nm}$ were used to grow single and multi-barrier RTD structures. The growth process was monitored using in-situ reflection high-energy electron diffraction (RHEED). After growth, the surface morphology of the films was determined using atomic force microscopy (AFM). Vertical $100 \mu \mathrm{m}$ diameter RTD devices were fabricated using standard lithographic meth- ods and reactive ion etching. Ti-Al was deposited and annealed at $\sim 450^{\circ} \mathrm{C}$ to form the $N$-type contacts to the top of the mesa and to the MOVPE $n^{+}$GaN layer. We measured current-voltage, $(I-V)$ and capacitance-voltage, $(C-V)$ characteristics for test RTD structures in the temperature range 4.2 to $400 \mathrm{~K}$.

\section{Results and discussion}

Figure 1 shows $I(V)$ curves measured at $300 \mathrm{~K}$ for one of these RTD samples. This particular sample had the following structure: a $2 \mathrm{~nm}$ AlN lower barrier, $2 \mathrm{~nm} \mathrm{GaN}$ quantum well, and $1 \mathrm{~nm}$ AlN upper barrier. In our experiment, the reverse bias corresponds to the negative potential and thus electron injection from the top contact. Curve $A_{1}$ in Fig. 1 is a sweep from 0 to $-8 \mathrm{~V}$ and shows a clear peak near $-6 \mathrm{~V}$; in the curve $B$ sweep from -8 to $+7 \mathrm{~V}$, no peak is visible in the reverse bias and the current is considerably smaller than on sweep $A_{1}$, particularly close to $0 \mathrm{~V}$. Curve $B$ shows a weak peak close to $+5 \mathrm{~V}$, which is absent on the subsequent down sweep $G$ from $+7 \mathrm{~V}$ to zero bias. Curves $A_{2}$ and $A_{3}$ plot $I(V)$ on subsequent sweeps from $0 \mathrm{~V}$ to high reverse bias and show a peak, though weaker and at a different bias position compared to the first upsweep $A_{1}$. Note that a sweep to high forward bias, followed by a sweep into the reverse bias, significantly increases the differential conductance $(G=d I / d V)$ around zero bias and in the reverse bias, compared with its value on a voltage sweep from the high reverse bias back to zero; see curve $B$ in Fig. 1 . The peak in $A$ tends to degrade further on subsequent sweeps into the reverse bias. These observations were accompanied by the weak dependence of the $I(V)$ on magnetic field applied parallel to the QW plane, and by the presence of large current discontinuities in the low temperature $I(V)$ curves. The latter effect is shown in Fig. 2, where solid line in $I(V)$ characteristics represents the current measured under the first voltage sweep from $-0.5 \mathrm{~V}$ to $+0.5 \mathrm{~V}$

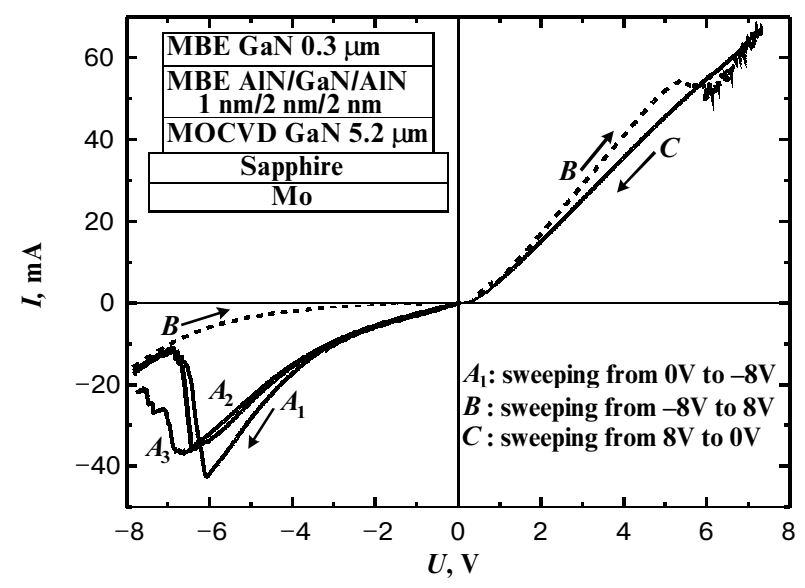

Fig. 1. Current-voltage characteristics of RTD device measured at $300 \mathrm{~K}$ under different voltage sweeps. 


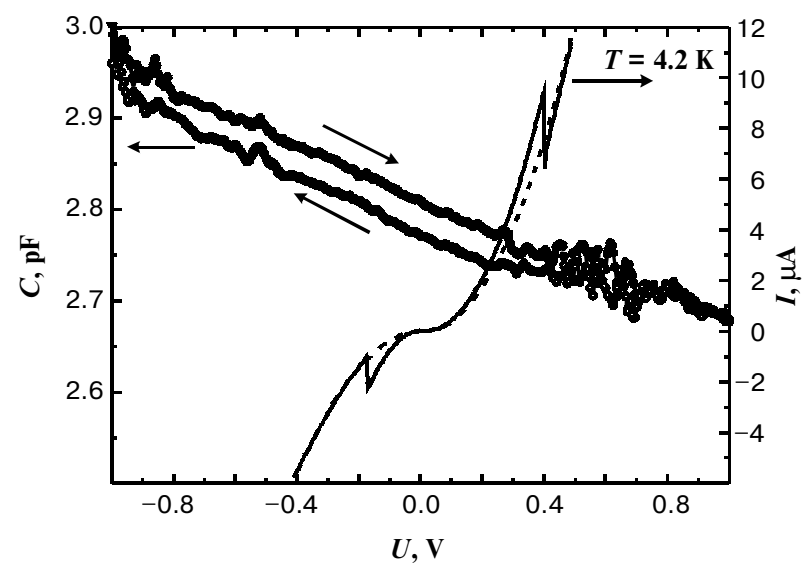

Fig. 2. Current-voltage (right axis) and capacitance-voltage (left axis) characteristics of RTD devices at $4.2 \mathrm{~K}$.

at $4.2 \mathrm{~K}$. At room temperature such low-voltage features are not easily observed.

To understand this complex behaviour, we must consider the effects of the polarisation fields and the influence of interface defects existing in this system. In wurtzite nitride semiconductors grown in the [0001] or [000-1] direction, spontaneous and piezo-electric polarisation is presented. Due to the large difference in the spontaneous polarisation between AlN and GaN, a huge electric field is formed in both the well and the barriers. This modifies considerably the potential profile of the double barrier structure. To understand the effect of polarization charges on the potential profile of our RTD structure, we performed self-consistent simulations of the conduction band profile using a model based on real-time tight-binding Green's functions [9]. From convergent beam electron diffraction studies of the sample we established that our MBE layers on GaN MOVPE templates are of Ga-polarity. Therefore, we have assumed alternating polarization charge densities of $\pm 3 \cdot 10^{13} \mathrm{~cm}^{-2}$ at the heterointerfaces for Ga polarity structure.

The results of the simulation are shown in Fig. 3. It is seen that the polarization charges produce a strong asymmetry of both the potential and charge profiles. A welldeveloped accumulation layer is formed in front of the first barrier adjacent to the substrate even at zero bias conditions (upper graph in Fig. 3). At the same time, an extended depletion region appears on the opposite side of the double barrier structure.

Under the reverse bias, the width of the depletion region decreases slowly and flat band conditions occur at $U \approx-2.6 \mathrm{~V}$. At this voltage, the ground quasi-bound state in the QW is already located below the Fermi level in the emitter (middle graph in Fig. 3). Thus, the first level in the QW crosses the Fermi level in the emitter under conditions when transparency of incoming barrier is much lower than that of outcoming barrier, i.e. under unsatisfactory conditions for resonant tunnelling. We therefore only expect to observe resonant tunnelling through the excited quasi-bound states in the $\mathrm{QW}$ with a further in-
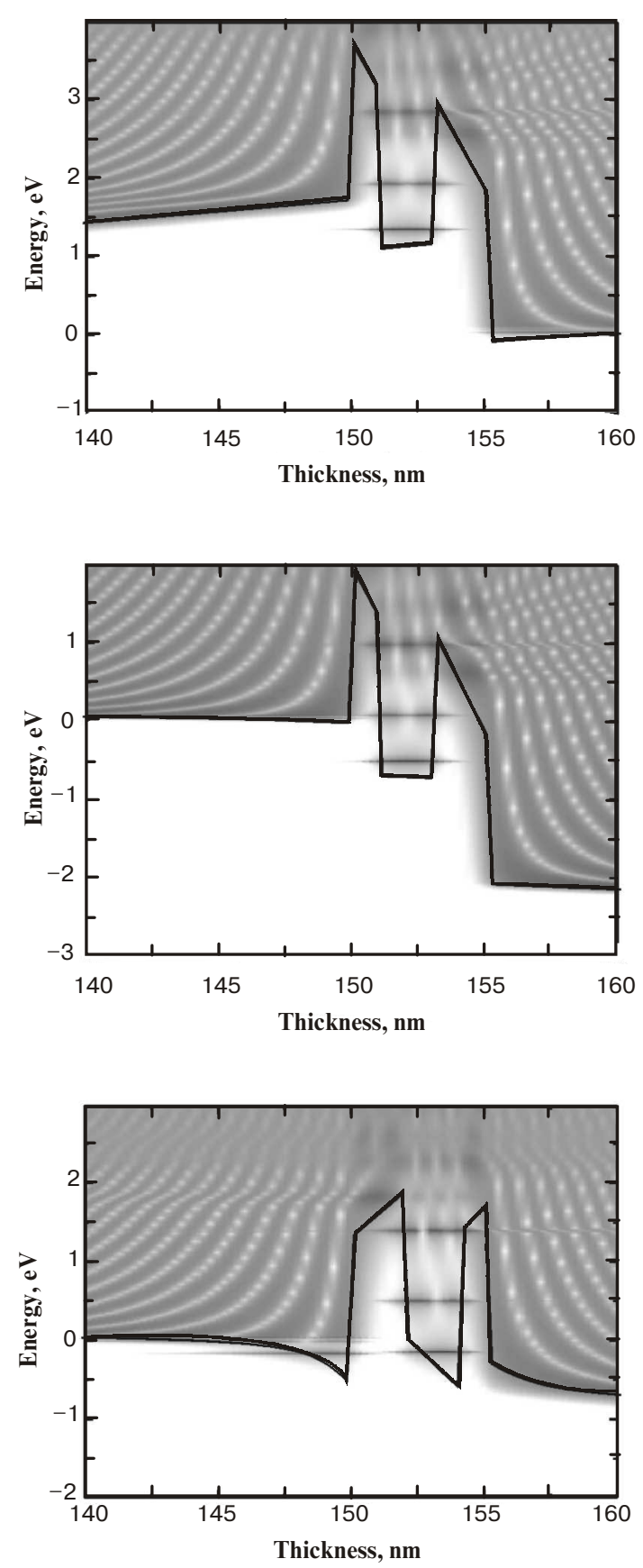

Fig. 3. Calculated local density of states and potential profile of RTD devices for different bias voltages: upper $-0 \mathrm{~V}$; middle reverse bias $-3.2 \mathrm{~V}$; lower - forward bias $+4 \mathrm{~V}$.

crease of the applied voltage. This conclusion is supported by simulation of $I(V)$ characteristic at room temperature shown in Fig. 4.

Under the forward bias, most of applied voltage drop occurs over depletion region on the collector side causing stronger depletion. The energy levels in the QW shift very slowly and even at $2 \mathrm{~V}$ the ground quasi-bound state in the QW remains higher than the Fermi level in the emitter. As a result, resonant tunnelling cannot be revealed in this forward voltage range and current discon- 
A.E. Belyaev et al.: Current instabilities in resonant tunnelling diodes based on ...

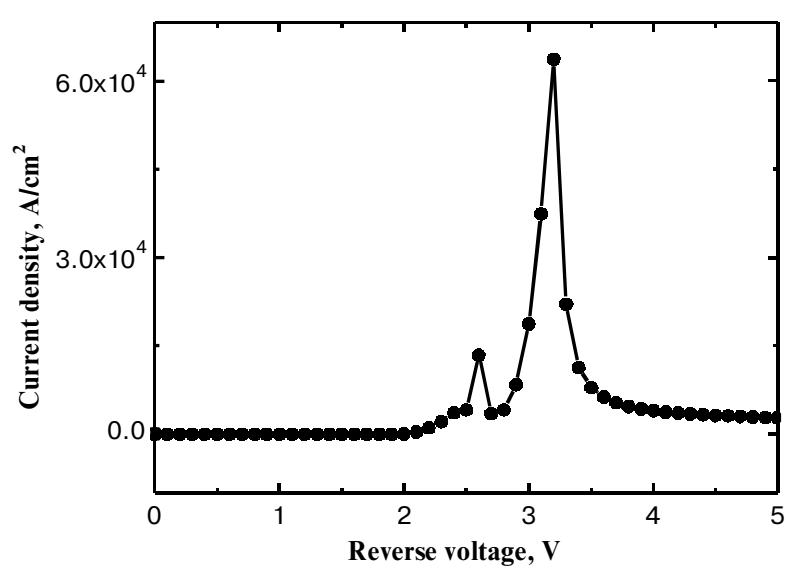

Fig. 4. Simulated current-voltage characteristics of RTD device $(T=300 \mathrm{~K}$, reverse bias $)$.

tinuities observed at low temperature and small bias cannot be prescribed to resonant tunnelling through the quantized subbands of the GaN QW.

The results of the simulation are supported by measurements of capacitance of the device presented in Fig.5. The capacitance curve is strongly anisotropic. The capacitance shows a local minimum around zero bias. Under reverse bias, the decreasing width of the depletion region on the left side of the structure shown in Fig. 3 is more significant than changes in 2DEG wave-function stand-off on the right side of the structure, which causes the rise in the capacitance as shown in Fig. 5. Under forward bias, the changes in capacitance are determined initially by the shift of 2DEG wave function standoff towards the barrier and then by extension of depletion region on opposite side of the double barrier structure. As shown in Fig. 5 there is good agreement between the capacitance measured at $300 \mathrm{~K}$ and the results of the theoretical calculations. In calculating the capacitance we

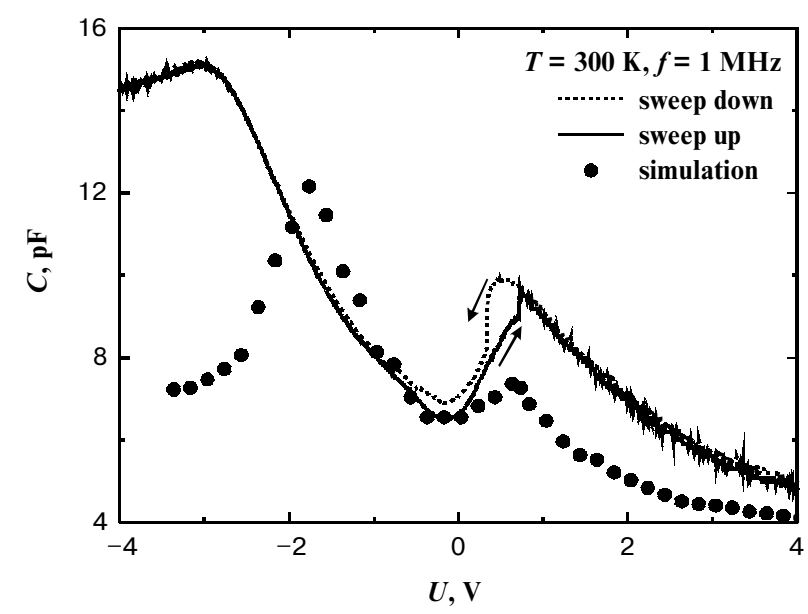

Fig. 5. Capacitance-voltage characteristics of RTD device at $300 \mathrm{~K}$ (solid and dashed lines are experimental data; solid circles are results of simulation). considered the double barrier structure as a simple flat capacitor, and mesa's area was used as fitting parameter. The capacitance of contacts can be neglected because of their magnitude is two orders greater.

From the consideration presented above, it is clear that the features observed in $I-V$ characteristics at low voltages $(-2 \mathrm{~V}$ to $+2 \mathrm{~V})$ cannot be ascribed to resonant tunnelling. It is more plausible to explain the current switches that are manifested at higher voltages as resonant tunnelling processes, but additional studies are needed to confirm this hypothesis. It is clear that the $I(V)$ curves of our RTD devices, particularly the existence of hysteresis and absence of any peak in the voltage sweeps from negative to positive bias, cannot be understood in terms of a simple model of electron resonant tunnelling through the quantized subbands of the GaN QW. This conclusion is supported by the weak dependence of the $I(V)$ characteristics on a magnetic field applied parallel to the plane of the quantum well, and by the presence of large discontinuities in the $I(V)$ curves measured at low temperatures $(4.2 \mathrm{~K})$.

Therefore, we attribute the strong hysteresis and the degradation of the peak in $I(V)$ to bias-dependent trapping of electrons, possibly in the GaN quantum well following tunnelling from the electron emitter layer. For example, the strong piezoelectric and spontaneous polarization effects in this material system could give rise to trapping of electrons at high reverse bias, leading to the low conductance observed on sweep $B$. The application of a large forward bias could release the electrons from the traps and restore the higher value of conductance around zero voltage and into negative bias. Charging-discharging processes are necessarily accompanied by changes in both vertical and lateral potential distributions. As a result non-linearities and instabilities will appear in the electrical characteristics of nitride semiconductor RTD devices. This may be due to rapid spatial or temporal variations in the distribution of mobile carriers and/or impurity/defect charges, fed with energy by a large electric field, i.e. the device is acting as a biasdependent switch. The charge storage should be the main reason of discrepancy between experimentally observed peak position (around $-6 \mathrm{~V}$ ) and one predicted by simulation (around $-3.2 \mathrm{~V}$ ) because the simulation has been performed without any charge accumulation in the QW.

The origin of traps is still under investigation and at present we can only speculate on possible sources. Perhaps the most probable source of traps is associated with the large concentration of interfacial states and dislocation states in our RTD structures. Carriers injected from the emitter can be trapped on these states, which cause an increase in the lateral non-uniformity of potential distribution and simultaneously lead to a redistribution of voltage drop throughout the structure. The latter should be the main reason, which stipulates the current switching. Under these conditions, it is very difficult to observe purely resonant tunnelling effects because the chargingrecharging processes mask this process. 


\section{A.E. Belyaev et al.: Current instabilities in resonant tunnelling diodes based on ...}

\section{Conclusions}

Therefore, to achieve stable operation of GaN/AlGaN RTDs, the device parameters need to be optimised. Firstly, it is necessary to decrease the asymmetry of both the potential profile and the charge distribution around the double barrier region. This can be achieved by an appropriate choice of barrier and well thickness and of the $\mathrm{Al}$ concentration in the barrier layers. It may also be important to minimize the density of defects related to dislocations, which can serve as traps for charge carriers and, thus, cause current instabilities.

\section{Acknowledgments}

This work was carried out in Nottingham University under EPSRC contract GR/R46465. The authors would also like to acknowledge the contributions of Mr. R.I. Dykeman, Mr. J.S. Chazuhan, Mr. D. Taylor and Mr. J.R. Middleton for device fabrication.

One of the authors (A.E.B) acknowledges the Royal Society and Deutsche Forschungsgemeinschaft for research grants. The work in FZJ was supported by the Office of Naval Research under Grant No. N00014-01-
1-0828 (monitored by Dr. Colin Wood) and by Deutsche Forschungsgemeinschaft (Project KL 1342). The work at V. Lashkaryov Institute of Semiconductor Physics in Kiev was supported by CRDF Project No. UE2-2439$\mathrm{KV}-02$.

\section{References}

1. J.W. Orton and C.T. Foxon // Reports in Progress in Physics, 61, p. 1 (1998).

2. S. Nakamura, M. Senoh, N. Iwasa and S.I. Nagahama // Jpn. J. Appl. Phys., 34, p. L797 (1995).

3. S. Nakamura, M. Senoh, S. Nagahama, N. Iwasa, T. Yamada, T. Matsushita, H. Kiyoku, Y. Sugimoto, T. Kozaki, H. Umemoto, M. Sano and K. Chocho // Jpn. J. Appl. Phys. Lett., 36, p. L1568 (1997).

4. C.H. Chen, S. Keller, G. Parish, R. Vetury, P. Kozodoy, E.L. Hu, S.P. Denbaars, U.K. Mishra and Y. Wu // Appl. Phys. Lett., 73, p. 3147 (1998).

5. A. Kikuchi, R. Bannai and K. Kishino // Phys. Stat. Sol. (a), 188, p. 187 (2001)

6. K. Kishino and A. Kikuchi // Phys. Stat. Sol. (a), 190, p. 23 (2002).

7. A. Kikuchi, R. Bannai, K. Kishino, C.M. Lee and J.I. Chyi // Appl. Phys. Lett., 81, p. 1729 (2002)

8. F. Sacconi, A. Di Carlo and P. Lugli // Phys. Stat. Sol. (a), 190, p. 295 (2002)

9. K.M. Indlekofer and J. Malindretos // http://www.fz-juelich.de/ isg/mbe/wingreen.html. 